\title{
Efficacy and Safety of Parecoxib in the Treatment of Acute Renal Colic: A Randomized Clinical Trial
}

\author{
Sidney Glina, Ronaldo Damiao, Joao Afif-Abdo, Carlos Francisco Santa Maria, Raúl Novoa, \\ Carlos Eurico Dornelles Cairoli, Dalia Wajsbrot, Gaston Araya
}

Hospital Ipiranga (SG), Sao Paulo; Hospital Universitario Pedro Ernesto (RD), Rio de Janeiro, Hospital Santa Cruz (JAA), Sao Paulo, Brazil; Clinica Anglo Americana (CFSM), Lima, Peru; Hospital del Salvador (RN), Santiago, Chile; Hospital Sao Lucas (CEDC), Porto Alegre, Brazil; Pfizer Inc. (DW), New York, NY, USA; Pfizer Central America \& Caribbean (GA), Heredia, Costa Rica

\begin{abstract}
Purpose: Although nonselective nonsteroidal anti-inflammatory drugs (nsNSAIDs) and opioids are effective treatments for acute renal colic, they are associated with adverse events (AEs). As cyclooxygenase-2 selective NSAIDs may provide a safer alternative, we compared the efficacy and safety of parecoxib versus an nsNSAID in subjects with acute renal colic.

Materials and Methods: Phase i.v., multicenter, double-blind, noninferiority, active-controlled study: 338 subjects with acute renal colic were randomized to parecoxib $40 \mathrm{mg}$ i.v. plus placebo $(\mathrm{n}=174)$ or ketoprofen $100 \mathrm{mg}$ i.v. plus placebo $(\mathrm{n}=164) .338$ subjects with acute renal colic were randomized to parecoxib $40 \mathrm{mg}$ i.v. $(\mathrm{n}=174)$ or ketoprofen $100 \mathrm{mg}$ i.v. $(\mathrm{n}=164)$ plus placebo. Subjects were evaluated 15, 30, 45, 60, 90 and 120 minutes after treatment start and 24 hours after discharge. Primary endpoint was the mean pain intensity difference (PID) at 30 minutes by visual analog scale (VAS) (per-protocol population). An ANCOVA model was used with treatment group, country, and baseline score as covariates. Noninferiority of parecoxib to ketoprofen was declared if the lower bound of the $95 \%$ confidence interval (CI) for the difference between the two groups excluded the pre-established margin of $10 \mathrm{~mm}$ for the primary endpoint.

Results: Baseline demographics were similar. The mean (SD) mPID30 min was 33.84 (24.61) and 35.16 (26.01) for parecoxib and ketoprofen, respectively. For treatment difference (parecoxib-ketoprofen) the lower bound of the $95 \% \mathrm{CI}$ was 6.53 . The mean change from baseline in VAS 30 minutes after study medication was $\sim 43$ mm; AEs were comparable between treatments.

Conclusions: Parecoxib is as effective as ketoprofen in the treatment of pain due to acute renal colic, is well tolerated and has a comparable safety profile.
\end{abstract}

Key words: parecoxib; ketoprofen; renal colic; lithiasis; pain; analgesia; COX-2 selective

Int Braz J Urol. 2011; 37: 697-705

\section{INTRODUCTION}

Acute renal colic or nephrolithiasis occurs when mineral or organic solids pass though the upper urinary tract and obstruct the urinary flow (1). It is a common, often recurrent condition with an annual incidence of 1-2 cases per 1000 and a lifetime risk that is greater in men than women (between 10-20\% and $3-5 \%$, respectively) (2). Patients usually present with a sudden onset of severe urinary pain that radiates from the flank to the groin and requires immediate treatment (within 30 minutes of the onset of symptoms) (1). In addition to severe pain, the main signs and symptoms of renal colic include nausea, 
vomiting, hypertension, swollen abdomen, fever and chills and hematuria.

Pain relief often takes the form of a nonselective, nonsteroidal anti-inflammatory drug (nsNSAID) or an opioid (1). Nonselective NSAIDs such as ketoprofen reduce pain and inflammation by inhibiting the cyclooxygenase (COX) enzyme (3). The COX enzyme exists in two distinct isoforms: COX1, the primary site of action for nsNSAIDs, is present in many tissues and is necessary for physiological (homeostatic) functions such as gastric mucosal protection and normal platelet aggregation $(4,5)$; COX2 is an inducible form of the COX enzyme and is expressed locally in inflamed tissues $(6,7)$. Although nsNSAIDs have been shown to be effective for acute and chronic pain relief, a number of adverse events (AEs) have been associated with their use. Common side effects from their use include rash, headaches, dizziness, drowsiness, abdominal pain, nausea, diarrhea, constipation and the retention of fluid.

Parecoxib, an injectable COX-2 selective NSAID, is currently the only available nonopioid analgesic and anti-inflammatory agent indicated for parenteral use that does not interfere with platelet aggregation $(8,9)$. As parecoxib is intended for the short-term treatment of acute pain it may, therefore, offer advantages versus nsNSAIDs in the treatment of acute renal colic; however, to date, no clinical studies have evaluated the use of parecoxib in acute pain due to renal colic.

The objective of this study was to compare the analgesic efficacy and safety of parecoxib $40 \mathrm{mg}$ intravenous (i.v.) administration versus ketoprofen $100 \mathrm{mg}$ i.v. to demonstrate noninferiority of parecoxib related to ketoprofen for reducing pain during an acute renal colic attack.

\section{MATERIALS AND METHODS}

\section{Study Population}

Subjects aged 18-65 years, with a confirmed diagnosis of renal colic either prior to or after randomization (as confirmed by radiography, i.v. pyelogram, helical computed tomography, magnetic resonance imaging, ultrasonography, or who had a diagnosis of renal colic confirmed by the subject's self-reported spontaneous elimination of the kidney stone at any time during the study) who presented with moderate to severe pain (baseline pain intensity [PI] score on a 100-mm visual analog scale [VAS] of $>50 \mathrm{~mm}$ and "moderate to severe" on a PI categorical scale) were eligible for inclusion in this study. All subjects provided written informed consent before entering the trial.

Subjects were excluded if they had significant renal or hepatic conditions (other than uncomplicated kidney stones), acute pain (other than renal colic), had been a recipient of a renal allograft, or were being treated for a urinary tract infection, pyelonephritis or clinical suspicion of such infection. In addition subjects were excluded if they had: a history of active peptic ulceration, active dyspepsia, gastrointestinal bleeding (i.e. Crohn's disease or ulcerative colitis), and an esophageal, gastric or duodenal ulcer within 1 month prior to the screening evaluation.

The study was conducted in accordance with the ethical principles outlined in the Declaration of Helsinki, International Conference on Harmonisation ( $\mathrm{ICH}$ ) Good Clinical Practice Guidelines and local regulatory requirements. The protocol was approved by the local institutional review boards and independent ethics committees and all subjects provided written informed consent. The ClinicalTrials.gov identifier is NCT00553605.

\section{Study Design}

This study was a phase i.v., multicenter, randomized, double-blind, double-dummy, comparative, active-controlled study to assess the analgesic activity and safety of i.v. doses of parecoxib $40 \mathrm{mg}$ relative to i.v. doses of ketoprofen $100 \mathrm{mg}$ for the treatment of renal colic in outpatients presenting at emergency room settings and was designed to show noninferiority of parecoxib related to ketoprofen.

Subjects were stratified by baseline PI into those with moderate or severe pain, according to the categorical PI scale. Using a computer-generated, block randomization schedule (block size 4), subjects were randomized 1:1 within each stratum and each study site to receive parecoxib $40 \mathrm{mg}$ i.v. (n $=174)$ plus placebo or ketoprofen $100 \mathrm{mg}$ i.v. $(\mathrm{n}=$ 164) plus placebo. To ensure both physicians and nurses were blinded to treatment, the randomiza- 
tion schedule was distributed in a sealed envelope to the pharmacist in charge of preparation and dispensing of the study drug. Subjects were evaluated at screening, initiation of drug infusion (time 0 ), and at $15,30,45,60,90$ and 120 minutes after study drug administration; follow-up was conducted 24 hours after discharge. If required, rescue medication (i.v. morphine) was allowed.

\section{Efficacy Assessments}

The primary efficacy variable was the mean pain intensity difference (PID) at 30 minutes after administration of study medication $\left(\mathrm{mPID}_{30 \mathrm{~min}}\right)$ assessed by VAS for the per-protocol (PP) population. The $\mathrm{mPID}_{30 \mathrm{~min}}$ for each subject is the sum of the PID at 15 minutes (VAS at baseline - VAS at $15 \mathrm{~min}$ utes) and at 30 minutes (VAS at baseline - VAS at 30 minutes), divided by two. This represents the mean reduction in PI across 30 minutes.

Secondary endpoints included: 1) PI assessed through VAS scores at all time points; 2) PID - change from baseline in VAS scores - at all time points; 3) mean PID at 120 minutes after the administration of study medication $\left(\mathrm{mPID}_{120 \mathrm{~min}}\right)$;

$\mathrm{mPID}_{120 \mathrm{~min}}=\frac{15\left(P I D_{15}+P I D_{30}+P I D_{45}+P I D_{60}\right)+30\left(P I D_{90}+P I D_{120}\right)}{120}$

4) response in PI (decrease of $>20 \mathrm{~mm}$ on the pain VAS score) at 30 minutes; 5) pain relief (PR) at 30 and 120 minutes; 6) sum of time interval weighted PR scores through 120 minutes (TOT$\left.\mathrm{PAR}_{120 \mathrm{~min}}\right)-$ TOTPAR $\left._{120 \mathrm{~min}}=30 P R_{30 \mathrm{~min}}+90 P R_{120 \mathrm{~min}} ; 7\right)$ subjects' global evaluation of study medication at 30 and 120 minutes, and at day 2;8) physician's global evaluation of study medication at 30 and 120 minutes, and at day 2; and 9) time to rescue medication up to 120 minutes.

The PP population was used for the analysis of the primary efficacy variable and included all subjects from the modified intent-to-treat (mITT) population who also satisfied the following criteria: 1) had no major pre-existing protocol violations; 2) received the appropriate dose of study medication; 3) had valid baseline (time 0), 15- and 30-minute VAS pain assessments according to the protocol; 4) did not take rescue medication during the first 30 minutes; and 5) had a confirmed diagnosis of nephrolithiasis or ureterolithiasis. The mITT population included all randomized subjects who took at least one dose of study drug and provided at least one postbaseline pain assessment. The mITT population was used for the analysis of all secondary efficacy endpoints.

\section{Safety Assessments}

The safety population included all subjects who were randomized and received at least one dose of study medication.

\section{Statistical Analysis}

The trial was intended to show noninferiority of parecoxib versus ketoprofen. Using a sample size of 168 subjects per group and assuming a standard deviation of $25 \mathrm{~mm}$ and a Type I error rate of 0.050 , the trial had $80 \%$ power to reject the null hypothesis (that parecoxib and ketoprofen were not equivalent) in favor of the alternative hypothesis of noninferiority. Noninferiority would have been declared for parecoxib if the lower bound of the two-sided 95\% confidence interval (CI) of the treatment difference (parecoxib - ketoprofen) was greater than - $10 \mathrm{~mm}$. The CI of the treatment difference was generated from the least squares means of an analysis of covariance (ANCOVA) model with terms for treatment, baseline VAS scores and country. All continuous variables were analyzed using this type of ANCOVA model. The Cochran-Mantel-Haenszel method with Ridits score (controlling for country) was used for the analysis of categorical variables; Kaplan-Meier techniques and log-rank tests were used for the analysis of the time to rescue medication.

\section{RESULTS}

\section{Subjects}

The study was conducted at 16 centers (emergency departments at orthopedic hospitals and general hospitals, and general practices) in Latin America (Brazil, Chile, Costa Rica, Ecuador, Honduras and Peru) between June 2007 and June 2009. Three hundred forty subjects were randomized to treatment, of which 338 received study medication and 333 (98.5\%) completed the study (Figure-1). A 
Figure 1 - Subject disposition.

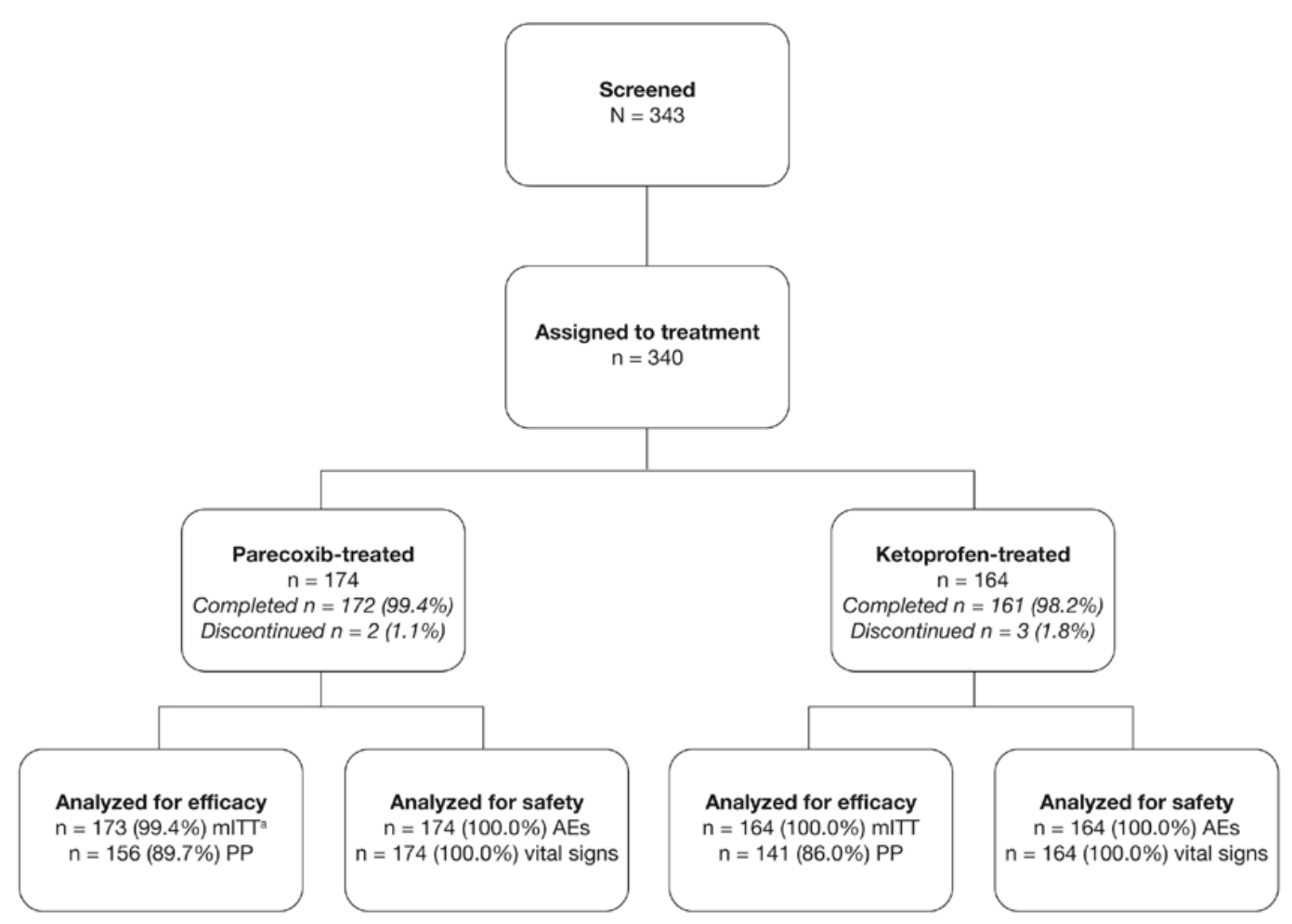

${ }^{a}$ One subject was excluded from the efficacy analysis (mITT) for the parecoxib group due to absence of any postbaseline assessment.

total of $10(5.7 \%)$ subjects from the parecoxib group and $14(8.5 \%)$ subjects from the ketoprofen group were excluded from the primary analysis because they did not have a confirmed diagnosis of renal colic. One subject in the parecoxib group and three subjects in the ketoprofen group discontinued the study because they did not meet the entrance criteria and one subject in the parecoxib group discontinued due to infusion problems (Figure-1). Demographics and clinical characteristics of subjects at randomization were similar in both treatment groups (Table-1). The majority of subjects were aged 18-64 years $(99.1 \%)$, $63.0 \%$ of subjects were male and $58.9 \%$ were white (Table-1). In addition, $60.3 \%$ and $57.3 \%$ of subjects in the parecoxib and ketoprofen groups, respectively, had severe pain at screening.

\section{Efficacy Results}

The noninferiority criterion was met for the primary efficacy endpoint, the $\mathrm{mPID}_{30 \mathrm{~min}}$. The $95 \%$
$\mathrm{CI}$ of the treatment difference (parecoxib - ketoprofen) was $-6.53 ; 4.30$ and it excluded the noninferiority margin of $-10 \mathrm{~mm}$ (Table-2). There were no significant differences observed between the treatment groups with regard to the secondary efficacy endpoints.

There were no significant differences observed between the two treatment groups with regard to pain VAS scores at any time (Figure-2).

The average $\mathrm{mPID}_{120 \mathrm{~min}}$ of both treatment groups was approximately $52 \mathrm{~mm}$ (SD of $\sim 26$ ) and $\sim 77 \%$ of subjects had a decrease of at least $20 \mathrm{~mm}$ on the pain VAS score at 30 minutes.

Although differences between treatment groups were not statistically significant, more subjects in the parecoxib group reported "a lot" or "complete" pain relief at minute $30: 61 \%$ of subjects in the parecoxib group and $55 \%$ of subjects in the ketoprofen group. The percentage of subjects who reported "a lot" or "complete" pain relief by minute 
Table 1 - Summary of baseline demographic characteristics.

\begin{tabular}{lcc}
\hline & $\begin{array}{c}\text { Parecoxib } \\
(\mathbf{n}=\mathbf{1 7 4})\end{array}$ & $\begin{array}{c}\text { Ketoprofen } \\
(\mathbf{n}=\mathbf{1 6 4})\end{array}$ \\
\hline Gender, number (\%) of subjects & $110(63.2)$ & $103(62.8)$ \\
Male & $64(36.8)$ & $61(37.2)$ \\
Female & & \\
Age, years & $38.6(10.3)$ & $40.1(12.1)$ \\
Mean (SD) & $16-69$ & $19-71$ \\
Range (minimum-maximum) & & $100(61.0)$ \\
Ethnicity, number (\%) of subjects & $99(56.9)$ & $3(1.8)$ \\
White & $2(1.1)$ & $3(1.8)$ \\
Black & $2(1.1)$ & $58(35.4)$ \\
Asian & $71(40.8)$ & $74.4(15.3)$ \\
Other & & $50-125$ \\
Weight, kg & $75.4(15.1)^{\mathrm{a}}$ & \\
Mean (SD) & $46-130^{\mathrm{a}}$ & $70(42.7)$ \\
Range (minimum-maximum) & & $94(57.3)$ \\
Categorical Pain Scale, number (\%) of subjects & $69(39.7)$ & \\
Moderate Pain & $105(60.3)$ & \\
Severe Pain & & \\
\hline
\end{tabular}

$n=$ number of subjects; $S D=$ standard deviation

${ }^{a} n=172$

Table 2 - Mean pain intensity difference at minute 30 by VAS ( $\mathrm{mm})$ for the PP population.

\begin{tabular}{lcc}
\hline & $\begin{array}{c}\text { Parecoxib } \\
(\mathbf{n = 1 5 6 )}\end{array}$ & $\begin{array}{c}\text { Ketoprofen } \\
(\mathbf{n = 1 4 1 )}\end{array}$ \\
\hline Mean (SD) & $33.84(24.61)$ & $35.16(26.01)$ \\
Median (minimum, maximum) & $35(-32,92)$ & $31(-43,98)$ \\
LS Mean (SE) & $34.147(3.35)$ & $35.266(3.46)$ \\
Parecoxib-ketoprofen & & \\
LS Mean (SE) & & \\
$95 \% \mathrm{CI}^{\mathrm{a}}$ & $-1.12(2.75)$ & \\
\hline
\end{tabular}

$n=$ number of subjects; $S D=$ standard deviation $; L S=$ least squares; $S E=$ standard error; $C I=$ confidence interval; VAS $=$ visual analog scale; $P P=$ per-protocol

${ }^{a}$ Based on ANCOVA model with terms for treatment group, country and baseline as covariates.

120 in the parecoxib and ketoprofen groups was $88 \%$ and $91 \%$ respectively.

Subjects treated with parecoxib reported a slightly higher mean $( \pm \mathrm{SD})$ score for TOTPAR ${ }_{120 \text { min }}$ than subjects in the ketoprofen group $(365.6 \pm 122.8$ vs. $355.0 \pm 126.1$, respectively); however, this difference was not statistically significant.

The percentage of subjects that assessed the medication as "good" or "excellent" at minute 30 , minute 120 and day 2 time points, was $81 \%, 86 \%$ 
Figure 2 - Mean $(S D)$ of the change from baseline in pain VAS $(\mathrm{mm})$ scores at all time points for the mITT population.

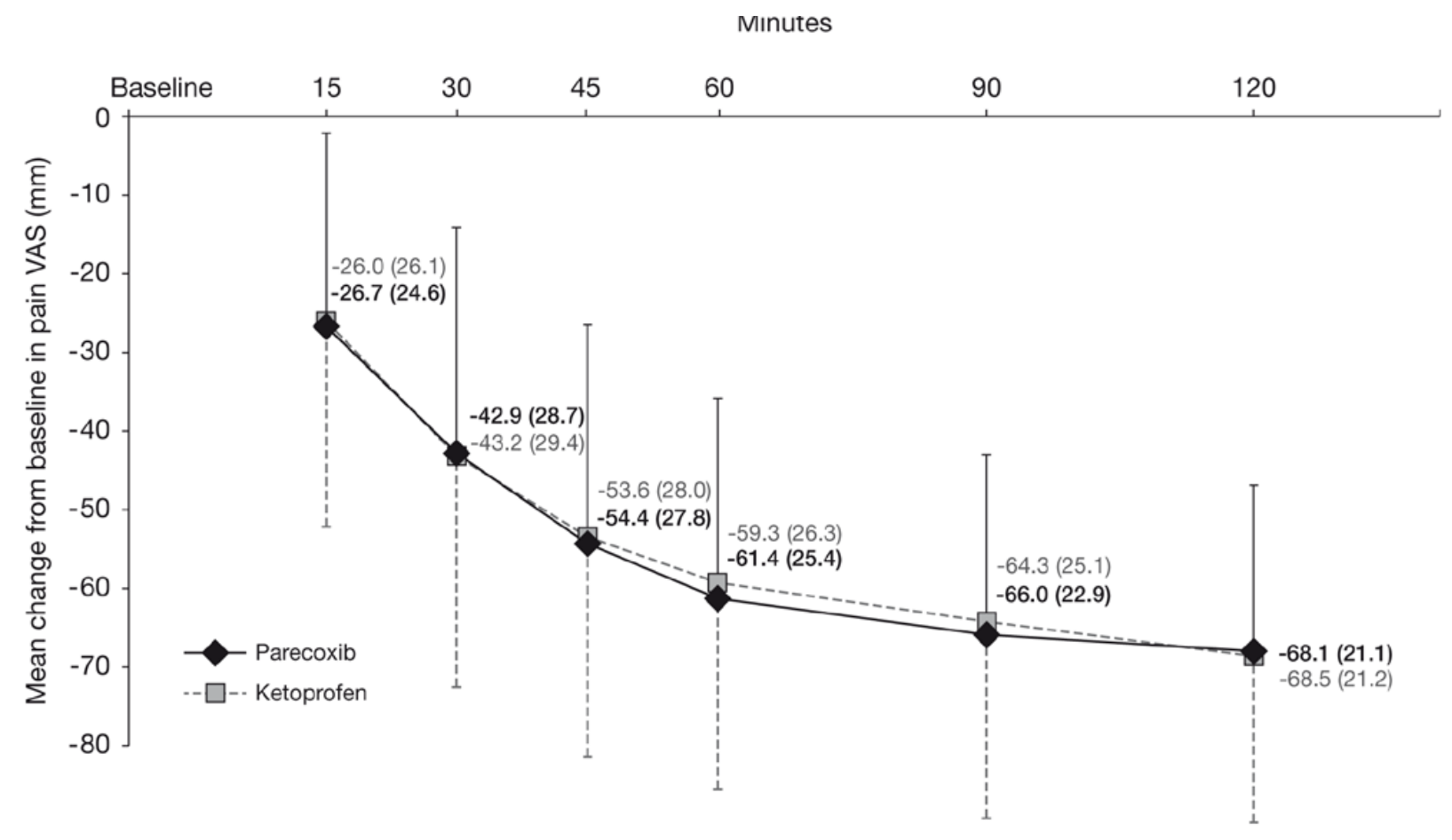

and $90 \%$ for the parecoxib group and $83 \%, 87 \%$ and $86 \%$ for the ketoprofen group, respectively. Again, no significant treatment differences were observed.

Similarly the physician's assessment of the medication was "good" or "excellent" at minute 30 , minute 120 and day 2 time points for $81 \%, 87 \%$ and $89 \%$ for the parecoxib group and $79 \%, 86 \%$ and $86 \%$ for the ketoprofen group, respectively.

The time to rescue medication was similar for both treatment groups. Twenty-six subjects took rescue medication up to 120 minutes after the administration of study medication in the parecoxib group compared with 25 subjects in the ketoprofen group. For both treatments, more than one-half of these subjects took the rescue medication within 60 minutes of dosing.

\section{Safety}

The incidence of AEs was similar for the two treatment groups. In the parecoxib group $25.9 \%$ of subjects experienced a total of 56 AEs; for the ke- toprofen group $28.0 \%$ of subjects experienced a total of 63 AEs. Overall, AEs were mainly mild or moderate in severity. A total of 16 subjects reported serious AEs; eight in each group (4.6\% in the parecoxib group and $4.9 \%$ in the ketoprofen group); none were considered by the investigator to be related to study drug and all resolved. No deaths were reported in either treatment group.

Pain (preferred terms pain, abdominal pain, renal colic and renal pain) was the most frequently reported $\mathrm{AE}$, experienced by $8 \%$ of subjects in the parecoxib group and $12.8 \%$ of subjects in the ketoprofen group (Table-3); again, none of these pain episodes was considered to be treatment-related. Although nausea was the second most frequently reported AE only two subjects in the parecoxib group and three subjects in the ketoprofen group experienced nausea that was considered to be treatment-related.

In terms of treatment-related AEs, dizziness was the most frequently reported. Furthermore, dizziness was more frequently reported for sub- 
Table 3 - Most frequently reported adverse events by preferred term.

\begin{tabular}{lcccc}
\hline Number (\%) of Subjects With Preferred Term AE & \multicolumn{2}{c}{$\begin{array}{c}\text { Parecoxib } \\
(\mathbf{n = 1 7 4 )}\end{array}$} & \multicolumn{2}{c}{$\begin{array}{c}\text { Ketoprofen } \\
(\mathbf{n}=\mathbf{1 6 4})\end{array}$} \\
\cline { 2 - 5 } & All Causality & Treatment-related & All Causality & Treatment-related \\
\hline Pain, abdominal pain, renal colic, or renal pain & $14(8.0)$ & 0 & $21(12.8)$ & 0 \\
Nausea & $7(4.0)$ & $2(1.1)$ & $5(3.0)$ & $3(1.8)$ \\
Dizziness & $7(4.0)$ & $5(2.9)$ & $2(1.2)$ & $1(0.6)$ \\
Vomiting & $4(2.3)$ & $1(0.6)$ & $5(3.0)$ & $2(1.2)$ \\
Headache & $3(1.7)$ & $2(1.1)$ & $4(2.4)$ & $3(1.8)$ \\
Flatulence & 0 & 0 & $4(2.4)$ & $3(1.8)$ \\
\hline
\end{tabular}

$A E=$ adverse event; $n=$ number of subjects

Table shows adverse events reported for more than $2 \%$ of subjects (all causality) in at least one treatment group.

jects treated with parecoxib (2.9\%) than ketoprofen $(0.6 \%)$. Worsening of renal colic was reported by $2.9 \%$ of subjects in the parecoxib group and by $4.3 \%$ of subjects in the ketoprofen group.

\section{DISCUSSION}

A common cause of acute severe pain, renal colic requires rapid medical attention, often prior to a diagnosis being made. Strong opioids such as morphine are commonly used in the treatment of pain associated with renal colic; however, although highly effective, opioids can cause a number of serious side effects in the central nervous system (i.e. dizziness, somnolence, respiratory depression, confusion and addiction), which may limit their use in the long-term.

Current evidence suggests that, like opioids, nsNSAIDs are efficacious in controlling the signs and symptoms of renal colic (1); those drugs reduce urinary system distension and the associated pain through the inhibition of prostaglandins (10). As nsNSAIDs lack the addiction risk and known side effects of opioids, they may be the preferred treatment choice for the majority of patients with acute pain due to renal colic.

The COX-2 selective NSAID parecoxib has also been used in the treatment of acute pain; however, unlike ketoprofen, parecoxib -another largely used drug to treat pain associated with renal colic- does not cause inhibition of platelet function $(11,12)$ and, thus, avoids the risk of bleeding. In contrast to certain parenteral NSAID formulations that require setting up a slow i.v. infusion, parecoxib can be injected rapidly and directly into a vein; a useful property in the busy emergency room setting.

Phillips and colleagues have previously evaluated the use of celecoxib, the only other currently approved COX-2 selective NSAID available for the management of acute renal colic (13). In this prospective, randomized, controlled clinical trial of 53 patients they found that there were no significant differences between celecoxib and placebo for either pain scores or narcotic requirements (13). However in our study, reporting here for the first time the use of parecoxib in this study population, and based on the findings of the primary analysis, parecoxib was shown to be noninferior to ketoprofen in the treatment of acute pain due to renal colic. In addition, there were no significant treatment differences between parecoxib and ketoprofen for any of the secondary efficacy endpoints.

Parecoxib was well-tolerated in this study and demonstrated a comparable safety profile to ketoprofen. Although there were no obvious safety concerns relating to the administration of either parecoxib or ketoprofen in this study, previous reports suggest that parecoxib is associated with fewer side effects than ketoprofen $(8,9)$. 
One of our main study limitations was that many patients with severe pain were unable to tolerate their pain for long enough to be able to read and sign the informed consent form prior to receiving pain-relief medication. As a result, many of these patients took additional medications and were, therefore, ineligible for enrollment in the study, which could have potentially created a selection bias.

\section{CONCLUSIONS}

Parecoxib is as effective as ketoprofen in the treatment of pain due to acute renal colic, is well tolerated and has a comparable safety profile.

\section{ACKNOWLEDGMENTS}

As the corresponding author, I can confirm that all authors had access to all the study data, took responsibility for the accuracy of the analysis, and had full authority concerning preparation of the manuscript and the decision to submit the manuscript for publication. This study was sponsored by Pfizer Inc. Editorial support was provided by L. Prevost, BSc, of PAREXEL, and was funded by Pfizer Inc.

We would also like to thank Dr Raymond Cheung for his invaluable input and support at the study design phase. In addition, we would also like to thank all the principal investigators who participated in this study (please see Appendix 1) and who have provided support and technical assistance during the development of this manuscript.

We can also confirm that results from this trial were presented in poster form at the Confederation of American Urology (CAU) meeting in Chile (September 8-11, 2010).

\section{DISCLOSURES}

Drs. Sidney Glina, Ronaldo Damiao, Joao Afif-Abdo, Carlos Francisco Santa Maria, Raul Novoa, Carlos Eurico Dornelles Cairoli do not have any conflicts of interest to disclose.

\section{ABBREVIATIONS:}

$\mathbf{A E}=$ adverse event

ANCOVA $=$ analysis of covariance

$\mathbf{C I}=$ confidence interval

COX $=$ cyclooxygenase

i.v. $=$ intravenous

mITT $=$ modified intent-to-treat

$\mathbf{n s}=$ nonselective

NSAID $=$ nonsteroidal anti-inflammatory drug

$\mathbf{P I}=$ pain intensity

$\mathbf{P I D}=$ pain intensity difference

$\mathbf{P P}=$ per protocol

$\mathbf{P R}=$ pain relief

SD $=$ standard deviation

VAS $=$ visual analog scale

\section{Appendix 1: Investigators}

Dr. Eduardo Berna Bertero, Brazil

Dr. Ronaldo Damiao, Brazil

Dr. Sidney Glina, Brazil

Dr. Carlos Eurico Dornelles Cairoli, Brazil

Dr. Eric Roger Wroclawski, Brazil

Dr. Walter J. Koff, Brazil

Dr. João Afif-Abdo, Brazil

Dr. Antonio Martins BRAZIL

\section{CONFLICT OF INTEREST}

Drs Dalia Wajsbrot and Gaston Araya are both currently full-time employees of Pfizer Inc.

\section{REFERENCES}

1. Holdgate A, Pollock T. Nonsteroidal anti-inflammatory drugs (NSAIDs) versus opioids for acute renal colic. Cochrane Database Syst Rev [Internet]. $2005 \mathrm{Apr}$ 18 [Accessed 2011 May 25]; (2). Available at: http:// www2.cochrane.org/reviews/en/ab004137.html

2. Clinical Knowledge Summaries. Renal colic - acute [Internet]. [s.1.]: National Institute for Health and Clinical Excellence; c2011. [Accessed 2010 November 1]. Available at: http://www.cks.nhs.uk/renal_colic_acute/view_whole_topic 
3. Vane JR: Inhibition of prostaglandin synthesis as a mechanism of action for aspirin-like drugs. Nat New Biol. 1971; 231: 232-5.

4. Gudis K, Sakamoto C: The role of cyclooxygenase in gastric mucosal protection. Dig Dis Sci. 2005; 50(Suppl 1): S16-23.

5. Brzozowski T, Konturek PC, Konturek SJ, Brzozowska I, Pawlik T: Role of prostaglandins in gastroprotection and gastric adaptation. J Physiol Pharmacol. 2005; 56(Suppl 5): 33-55.

6. Seibert K, Zhang Y, Leahy K, Hauser S, Masferrer J, Perkins W, et al.: Pharmacological and biochemical demonstration of the role of cyclooxygenase 2 in inflammation and pain. Proc Natl Acad Sci U S A. 1994; 91: 12013-7.

7. Otto JC, Smith WL: Prostaglandin endoperoxide synthases-1 and -2. J Lipid Mediat Cell Signal. 1995; 12: 139-56.
8. Emery P: Clinical implications of selective cyclooxygenase-2 inhibition. Scand J Rheumatol Suppl. 1996; 102: 23-8.

9. Simon LS: Nonsteroidal antiinflammatory drugs and their effects. J Clin Rheumatol. 1996; 2: 135-40.

10. Tiselius HG, Ackermann D, Alken P, Buck C, Conort P, Gallucci M, et al.: Guidelines on urolithiasis. Eur Urol. 2001; 40: 362-71.

11. Noveck R, Laurent A, Kuss M, Talwalker S, Hubbard RC: Parecoxib sodium does not impair platelet function in healthy elderly and non-elderly individuals: Two randomized, controlled trials. Clin Drug Invest. 2001; 21: 465-76.

12. Munsterhjelm E, Niemi TT, Ylikorkala O, Neuvonen PJ, Rosenberg PH: Influence on platelet aggregation of i.v. parecoxib and acetaminophen in healthy volunteers. Br J Anaesth. 2006; 97: 226-31.

13. Phillips E, Hinck B, Pedro R, Makhlouf A, Kriedberg $\mathrm{C}$, Hendlin $\mathrm{K}$, et al:: Celecoxib in the management of acute renal colic: a randomized controlled clinical trial. Urology. 2009; 74: 994-9.

Submitted for publication:

December 20, 2010

\author{
Correspondence address: \\ Dr. Sidney Glina \\ Rua Cincinato Braga 37 / 102 \\ São Paulo, SP, 01333-011, Brazil \\ Fax: +55 11 3515-7900 \\ E-mail: glinas@terra.com.br
}

